\title{
Trinity of Genome on the Basis of DNA-Energy, DNA-Particle and DNA-Information: A Prokariotic Cell
}

\section{N. F. Kuznetsova}

All-Russian Research Institute of Forest Genetic, Breeding and Biotechnology, Voronezh, Russia

Email address:

ilgis@lesgen.vrn.ru

To cite this article:

N. F. Kuznetsova. Trinity of Genome on the Basis of DNA-Energy, DNA-Particle and DNA-Information: A Prokariotic Cell. European Journal of Biophysics. Vol. 4, No. 1, 2016, pp. 1-7. doi: 10.11648/j.ejb.20160401.11

\begin{abstract}
The study aim is the creation of the tridimensional corpuscular-wave model of prokaryotic cell, and the justification of the trinity principle of energy, matter and information on the example of a genome of prokaryotes. Analysis was carried out at the junction of molecular biology, biochemistry, biophysics, cell biology and physics (common and quantum). It is shown that during cell cycle on the basis of DNA molecule (particle and wave) operate independently and interconnected three complex genetic systems - energetic (DNA-energy), corpuscular (DNA-particle) and informational (DNA-information). Each of them is characterized by the different nature, mechanism action and functional predestination. DNA-energy and DNAinformation have the wave nature, DNA-particle - the corpuscular ones. DNA-energy associates with the work of biological harmonic oscillator, which possesses the energy and is able to lower the entropy of cell, DNA-particle forms a protein composition of cell, and DNA-information defines its species diversity. The main conclusion is that the life is realized not in one, as is accepted at the present time, and in three non-dividing and non-merging planes. Due to trinity principle a prokaryotic organisms in the thermodynamic, structural and informative relation become higher against an inanimate matter and such qualities as orderliness, particular chemical composition and species-specific organization are got.
\end{abstract}

Keywords: Prokaryotic Cell, DNA-Wave, DNA-Particle, Energetic System, Corpuscular System, Informational System

\section{Introduction}

Now it is proved, that a DNA molecule is a bearer of genetic (corpuscular) information. Particular protein composition of cell forms by means of a complex matrix process, which is carried out by the complementary principle in three stages - a replication, a transcription and a translation. As known, a biosynthesis of DNA, RNA and proteins are the endergonic reactions. Though a sources of energy for these processes are still a subject of discussions.

All living cells are characterized by an ordered structure. In the words of Schroedinger [1], "a life - is orderly and regular behavior of matter... which is supported by all the time." According to the second law of thermodynamics all spontaneously proceeding processes should go in the balance direction. Different opinions to the problem the relations of thermodynamics and biology take place - from full negation, applicability and partial spread on separate functional systems. Until today, a life as the anti-entropic process and a nature of the orderliness of cell systems are remained the controversial questions in biology.
As known, the prokaryotic organisms are characterized by not only orderliness, but also species heterogeneity. In the middle of the last century appeared the number of work, connected with the use of information theory in biology [2, 3]. The attempts to identify the information with the orderliness and anti-entropy processes were not conformed [4-6]. Korogodin [5, 6] defined the information as "guide to action", as a set of methods and devices, which can be used by organisms for the implement targeted actions. It should be noted that the elaboration of information theory in many respects associated with development of radio engineering as a science. Therefore search of similar systems into the cells is also the main task of this research.

In previous work [7], we have shown that, firstly, in the interphase period the DNA molecule should exist in the form of both particles and waves simultaneously (on the assumption of DNA electrochemical structure and the mitosis mechanism), and, secondly, a cell is a quantummechanical system, an open and closed system and an equilibrium system obeying the fundamental lows of physics, including the lows of thermodynamics. This work is a continuation of research of cell biology related with its wave 
nature.

As the model object we have taken a prokaryotic cell. All prokaryotes are the highly orderly living systems in comparison with the inanimate matter [8-14]. At the same time they are less developed and less complex integrity than the eukaryotic organisms. In the article the tri-plane corpuscular-wave model of a cell and the principle of trinity of energy, matter and information on the example of the genome of prokaryotic cell are discussed. According to this principle the cell life is carried out in three non-dividing and non-merging planes - energetic (on the basis of DNA-waveenergy or DNA-energy), corpuscular (on the basis of DNA particle) and informational (on the basis of DNA-waveinformation or DNA-information). Despite the fact, that energetic, substantial and informational components of genome are the equal in their significance, the central place among them undoubtedly belongs to the DNA-energy.

\section{Energetic System (DNA-Energy)}

A number of important consequences from DNA wave nature follow. Firstly, an oscillating DNA or a plurality of oscillating nucleotides of the dipolar structure [15] should create an electromagnetic field with two zones of action: the close-range (changing as $1 / \mathrm{r}^{2}, 1 / \mathrm{r}^{3}$ ) and far-range (changing as $1 / \mathrm{r}$ ) ones. At first this field was described by Gurvich [16], who called it a biofield. Secondly, a DNA-wave presents two waves are moving along the different threads of circular chromosome, therefore a DNA-wave is two standing waves. Thirdly, the cell together with the oscillating DNA - is a physical system - the quantum harmonic oscillator, which is both a physical and biological body simultaneously. Fourthly, a cell, as well as any oscillatory system, should obeys the thermodynamics laws, possess the energy and have the certain number of common quantum-mechanical features. Fifthly, the energetics of prokaryotic cells is provided by a single internal source (biological harmonic oscillator), one or several external sources (fermentation, photosynthesis, respiration), a centrifugal energy stream (on the basis of processing) and centripetal energy stream (in the system “ATP-ADP”).

Mazia [17] regarded the loss of ability to a cell reproduction as a synonym of its death. According to our understanding, a cell division is a secondary phenomenon in relation to the work of oscillator as the autonomous internal source of energy. This structure occupies the central place in energetic system of cell. It must be emphasized, that only in prokaryotes a cell together with the oscillating DNA is at the same time the source of energy, the cell and the independent organism.

By Hegel [18] the biological harmonic oscillator as regards to a cell is a structure of «an sich» and «für sich». Its energy can be two types - the total energy and free ones. A total energy consists of kinetic and potential energy, and characterizes an energetic potential of organism in a concrete period of time. A free energy is liberated during transition of a DNA-wave to a lower energy level and enters (once in a cell cycle) into a cell. The undamped oscillations on macromolecule at the expense periodically filling a cell by free energy are supported. The fact that confirms the existence in prokaryotic cells of internal source of energy is the ability of $E$. coli to a long-term cultivation under the conditions of substrate starvation [19]. During starvation a divisions of bacteria occur, but they are strongly suppressed. I.e. E. coli for normal vital function is undoubtedly required entering of energy from the outside.

As was mentioned above a prokaryotic cell is the open system and constantly exchanges with the environment of energy and substance. Prokaryotes have a variety of way of extracting and utilization the energy by means of complex mechanisms of fermentation, photosynthesis and respiration and covering it into chemical energy [11-14]. The majority of species receive energy by means of anaerobic fermentation a less effective way of biological oxidation. A supply of nutrients is carried out with the participation of biofield forces which act inside the cell and beyond. The energy, liberated during bacterial fermentation, passes in the energy of ATP chemical bonds and is used for replenishment of an energy balance of cell in the ATP-ADP system as the centripetal energy stream [7] and for performance of different works (chemical, mechanical, etc.).

Prokaryotes are very small organisms, and the smaller the organism, the higher the relative value of its surface in comparison with the volume [20]. Their cells have a relative an enormous surface as regards to its volume. Therefore a rigid cellular wall is the main way of protection of cell from constantly changing external environment. This wall also provides the reliability of work of a biological harmonic oscillator.

The oscillator of all prokaryotes has a rather simple structure. It is a single circular chromosome, which on both sides is attached to the plasmatic membrane. The most part of inner energy a cell is utilized by the several channels (fig. 1): 1) a replication; 2) a transcription and translation; 3) a cell growth; 4) a cytotomy; 5) a restitution of ATP from ADP; and 7) a bioluminescence. As the efficiency of cell systems can not be equal to $100 \%$, a part of the free energy is always lost.

A cell the most part of own life is in the main equilibrium state. In the equilibrium a cell has a zero-point energy, below which the energy of DNA-wave cannot be. Q-factor of prokaryotic oscillator is not so great, proceed from the fact that the nucleus is absent and a lot of energy is spent on a friction. The stability of zero level is supported thanks to a periodic transition of a macromolecule in the excited state (from $0^{\text {th }}$ on the $1^{\text {st }}$ energy level) at the conditions of higher intracellular pressure. We think that the pressure jump takes place in the moment, when growth of cell ceases, because it on the outside is surrounded by a rigid wall. As consequence, a synthesis of biomolecules occurs during some time into a fixed volume. The excited state of the macromolecule is unstable. A return to the main equilibrium state of DNAwave is associated with its radiation and two quantum of free energy (by one from each the standing wave) enters into a 
cell. At this time a mother cell is filled up the energy and two highly ordered processes are initiated, the end result of which are the formation of two daughter cells.

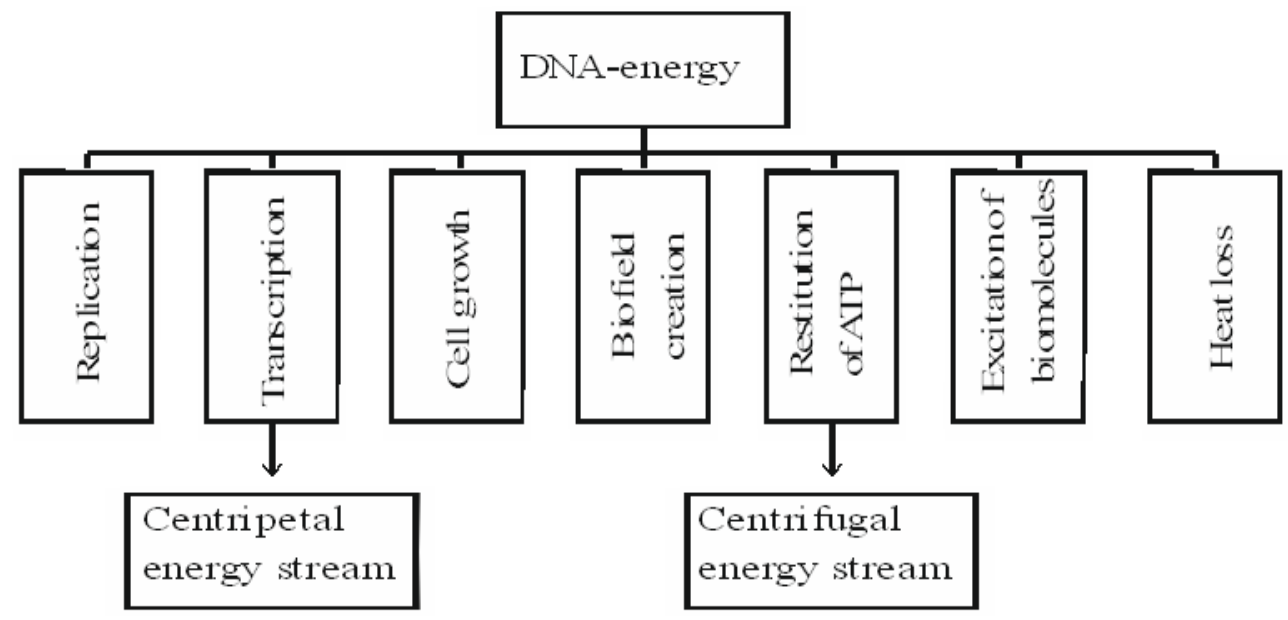

Figure 1. The channels of utilization of free energy from biological harmonic oscillator of prokaryotic cell.

In conclusion, it should be emphasized that an energetics of prokaryotic cell is provided by both the internal and external energy sources. The basis of energetic plane is biological harmonic oscillator, which is a biological and physical body simultaneously. We assume that energy of biological harmonic oscillator, external sources, centripetal and centrifugal energy streams and a biofield forces can be those factors thanks to which the cell overcomes a chaosogeneity of environment, creates an internal orderliness, provide the most intracellular reactions (replication, transcription, etc.) by energy.

The action of energetic system is limited by the zone of biofield, the forces of which extend on a cell and the nearby layer of environment. In all prokaryotes, the principle of organization and function of the genome in this respect is the same. DNA-wave for maintenance the viability of cells in the equilibrium state must periodically lifts from the $0^{\text {th }}$ to the $1^{\text {st }}$ energy level (an excited state). The free energy, which is liberated during returning of macromolecule to the main state, goes by two streams into a cell. In this time a cell is replenished by the energy. This energy initiates simultaneous two biophysical and biochemical processes, leading to a doubling of the DNA and the formation two new cells.

\section{Corpuscular System (DNA-Particle)}

All microorganisms are characterized the specific chemical composition, which has no analogue in the inanimate matter. The main intracellular components are proteins, which take part in the formation of all enzyme systems and constitutional proteins. The information about a molecular (corpuscular) cell body laid down in the chemical formula of DNA-particle, or in the nucleotide sequence of the only circular chromosome. The biosynthesis of protein is a complex matrix process, which occurs in three stages - a replication, a transcription and a translation [13, 14, 21]. Corpuscular-wave mechanisms of these processes are described in detail in the work [7]. Here we pay attention only to the questions connected with their energetics.

Beginning of formation of cell molecular composition is a DNA-particle doubling on complementary basis. During replication genetic state of macromolecule is change from 1 DNA-particle + 1 DNA-wave to 2 DNA-particle + 1 DNAwave. The process of replication, transcription and translation can be presented as the following sequence of events.

Replication. Replication is one of the most energetically dependent biochemical reactions. The analysis has shown that the chief role in initiation and realization of this process belongs to a biological harmonic oscillator. It energy, which is liberated during DNA-wave radiation, activates two molecules of DNA-polymerase. The activated DNA-polymerase, which have received the energy and the charge, are attracted to the counter waves of ring chromosome and begin to diverge from the certain point of DNA-particle (ORI) in opposite sides with the speed of waves carrying them. A negatively charged enzyme breaks the electric balance within the "enzyme-macromolecule" association. This leads to the local divergence of DNA threads and the formation of two replication forks. By course of wave motion a DNA-polymerase catalyzes the reaction of joining to each nucleotide of its complementary pair and, carrying out, thus, the DNA biosynthesis in a $5^{\prime}$ $\rightarrow 3^{\prime}$ direction. During replication the enzymes gradually lose own energy. After all two inactive enzymes (after the collision) turn into the cell. The wave nature of process ensures the same speed and high accuracy of replication which be equal to about $30 \mu \mathrm{m}$ in a minute [22] and one error on $10^{9}$ pairs of bases [23].

Transcription. The transcription is the next stage by a transfer, firstly, the corpuscular information from DNAparticle to RNA-particle, and, secondly, a energy from biological harmonic oscillator to the chemical bonds of different RNA molecules. Three types RNA (mRNA, rRNA and tRNA) take part in formation of a molecular body of cell. All types of RNA molecules synthesized on the DNA- 
particle matrix according by the complementary principle. In prokaryotes free energy liberated during DNA radiation activates two enzymes (DNA-polymerase and RNApolymerase), and they processes of replication and transcription processes proceed simultaneously.

The corpuscular-wave mechanisms of replication and transcription are in many respects analogous. During DNAwave radiation a molecules of RNA-polymerase receive the energy. Activated enzyme is attracted only to one thread of DNA-particle, forming with it a stable connection in the certain places - the promoters. The electric balance of charges within the "enzyme-macromolecule" association, due to the bond polarity and the asymmetry of enzyme structure, is broken. This causes a reduction of hydrogen connections and a local divergence of the threads of DNA. Promoters are located before an initiation point of transcription, and by a specific nucleotide composition and size are characterized. During transcription (elongation stage) the enzyme molecules, moving with DNA-wave along a copied region, untwist a double spiral DNA-particle, gradually expend own energy on RNA biosynthesis and build the new RNA molecules on a counter wave of the DNA-wave. After inactivation and collisions with a termination region the RNA-polymerase molecules are separated from the DNA-particle and pass into a cell.

All newly formed RNA undergo a post-transcriptional processing, or the ordered process of RNA disintegration, leading to the transformation of primary heavy RNA transcripts in functional mRNA, tRNA and rRNA. We assume that the processing reminds such physical phenomenon as an exponential disintegration, analogous to the process of heavy nuclei $\alpha$-disintegration, disintegration $\mathrm{U}^{238}$, etc. Its essence: at the spontaneous division of particles the probability of the disintegration never changes, therefore by the end of the first half of the disintegration period there remains $1 / 2$ particles, the second $-1 / 4$, etc. In cell cycle a process of spontaneous disintegration, which extended in time and goes with the free energy liberation, presents the centrifugal energy steam. Energy of this stream is used on the energetic supply of protein biosynthesis.

Translation. As has been said, the information about the chemical composition of proteins laid down in the nucleotide sequence of DNA-particle. During transcription this information has been transferred in the nucleotide sequence of mRNA-particle. Translation is the last stage connected with a formation of cell molecular body, or the process of information decoding in the amino acid sequence of proteins. We think that all types of RNA mRNA, rRNA and tRNA in the biosynthesis of protein are involved. The mRNA serves as a matrix, from which the amino acid sequence of proteins is read out. tRNA carry out the transport function and reading device. rRNA, mRNA and tRNA are like an "energy reservoir", which during post-transcriptional processing is gradually released. Processing or exponential disintegration of RNA molecules goes with allocation of free energy and provides the reaction of protein biosynthesis by energy. The joint work of mRNA, rRNA and tRNA is organized so, that before each division the quantity of protein in a cells doubles.

To summarize the information about a DNA-particle, it should be mention that a zone action of the corpuscular component of genome, as the sphere of DNA-particle action, is limited to framework of cell. Firstly, the DNA molecule is situated into a cell cavity. Secondly, the corpuscular-wave mechanisms of replication, transcription and translation are realized only inside a cell. Thirdly, main part of protein molecules after biosynthesis remains within a cell, due to a semi-permeability of plasmatic membrane.

Corpuscular and energetic components of genome are closely connected with each other. All species of prokaryotes for creation of a species-specific molecular composition use the same universal mechanisms - a replication, a transcription, a translation. This complex, high degree of precision matrix processes occurs in each cell, until a species exists. Biological harmonic oscillator takes part in the biosynthesis of DNA and RNA as: 1) a source of biofield; 2) a source of total and free energy; and 3) a standing DNAwave as means of transport. From three universal mechanisms only translation is not connected directly with the energetic system of genome. Though, indirect connection between them can be traced. Namely, the energetics of translation process is carried out at the expense of energy, which is liberated during processing of pre-rRNA precursors, synthesized during transcription due to the energy from an internal source - a biological harmonic oscillator.

\section{Informational System (DNA-Information)}

A living matter is characterized by not only the orderliness, but also species-specific organization. During tens and hundreds millions years a shape of prokaryotic organisms (cocci, rod-shaped, convoluted, branching) and their lifestyle species are transmitted from generation to generation and preserve its identity. According to Fox and Dose [24] a modern cyanobacteria is practically unchanged as its Precambrian ancestors. A cell after removal of outer wall by a lysozyme enzyme soon generates the same new envelope [10]. Cell systems, separated by a quartz glass, are capable to exchange by the regulatory information [25].

The organized systems are distinguished from the ordered ones [26]. It is considered that an organization is the complexity, which is endowed with function and information $[27,28]$. According to Wiener "Information is information, not matter and not energy" [28]. Between the initial information and the final objects should be present three types of systems [6]. Class A objects are the bearers of initial information. Their existence is not dependent from the class $\mathrm{B}$ and $\mathrm{C}$ objects. Class B - are the objects, on the basis of which information can be created. Class C - are the objects, for which information is needed. Class $\mathrm{B}$ devices are primary, and class $\mathrm{C}$ devices are secondary in relation to the information of class A. 
The nature of species specificity, intracellular mechanisms of a coding, storage and decoding of information has long been the subject of discussions among the scientists [5, 2729]. They, as rule, a source of genetic information associated only with the nucleotide sequence of DNA-particle. However, if it is laid and stored in a DNA-particle structure, it is not clear how over time this information is protected from degeneration. During numerous divisions a part of information certainly should be lost as a result of noises and hindrances. The mechanisms of its re-establishment are not known. Something like mechanistic methods of storage of the redundant information inside a cell are also not found. Moreover it is impossible to explain the of species organization as the DNA-energy function, because all prokaryotes have the same energy potential $\left(0^{\text {th }}-\right.$ the main and $1^{\text {st }}$ - the excited level). Therefore the species heterogeneity is not the function both a DNA-particle and DNA-energy. It will be once more plane of organization of the genome - the informational system, which acts on the basis of DNA-wave and is the part of genetic system. Under the informational system we shall understand the set of intracellular ways and devices that can be used for a construction of special intracellular operator - a DNAinformation (object class B) and a cell for the formation of which this species information is needed (object class C). The main task of the informational system consists in the creation in a space and a time of the certain directivity of matter and energy motion, which is manifested in the form of a species variability of individuals.

As known, the information can exist only being fixed on the some physical bearer. We paid attention to the fact, that a chromosome and DNA-particle of prokaryotic cell by a shape and inner structure very remind the frame antenna of Contrawound Toroidal Helical Antenna (CTHE) type. The CTHE antennas are characterized by a high Q-factor, a narrow bandwidth for the selected frequency, a weak influence of environment on the input impedance, a higher sensibility to magnetic component of electromagnetic field. It is intended for uninterrupted signal reception of a certain frequency on mobile objects. Constructional peculiarity of these antennas consists that at them two spirally twisted conductors as a frame are used. These conductors interconnect by means of few clamps and in the place of intersection are not incorporated. A hoop is the optimal form of frame antennas. The bearers of antenna for communication stability during movement should have a rigid construction.

Prokaryotes also are the mobile organisms and are surrounded outside by the dense cell walls, which give them rigidity. A chromosome attaches to the plasmatic membrane and presents a continuous circular cord (or frame microantenna). DNA-particle consists of two spirally twisted polynucleotide chains (or two spirally twisted conductors), connected by a hydrogen bonds (or a great number of clamps). The ends of each DNA thread are linked with a plasmatic membrane and are not joined among themselves. Though the similarity of their constructions is evident, however, the technical characteristics of the "prokaryotic STHE" in comparison even with the advanced Contrawound Toroidal Helical Antenna are more perfect. It should be said, that a micro-antenna of every individuals must be configured on the receiving only own species informational signal. Therefore various species have a different technical characteristics of "prokaryotic STHE" in depend of the diameter of circular chromosome, chemical composition of DNA-particle, frequency of DNA-wave oscillations, etc.

Having analyzed a cell cycle we concluded that a "prokaryotic STHE" may realizes the reception of informational signal only once in cycle, namely, during splitting of doubled DNA (a particle and a wave) (fig. 2). During divergence two newly formed mesosoma carry away behind themselves the daughter chromosomes. At beginning the doubled DNA is separated in the mesosoma area, afterwards the binding portion between them becomes less, and eventually two circular chromosomes remain connected only at the top of rings. The inclination angle of plane of a circular chromosome concerning of Earth surface is gradually changed. We think that in this moment two daughter "prokaryotic STHE" are automatic configured on the frequency of certain information signal. Therefore the logical question is, on what informational signal is configured the "prokaryotic STHE" antenna? We know nothing about the objects of class A, or the bearers of initial information.

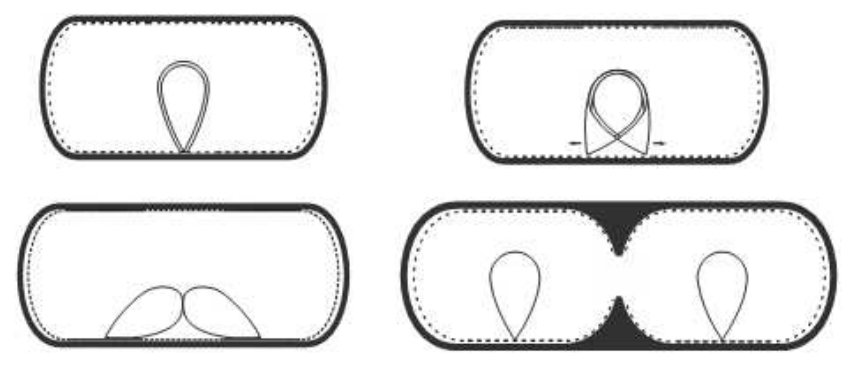

Figure 2. The scheme shows how during cell division a "prokaryotic Contrawound Toroidal Helical Antenna" can be configured on the reception of species information signal.

The search of theories and hypotheses for an explanation of species heterogeneity of microorganisms led us to the concept of cosmic organization of life. Vernadsky [30], the founder of the given doctrine, has described a life as the cosmic phenomenon. Chizhevsky [31] wrote, "Modern natural science has unusually widely moved apart the notion about the environment limits, having included in it the cosmic bodies, sending to us the electromagnetic waves and streams of particles". He suggested that on the Earth together with the cosmic streams comes a particular bioactive radiation, which was named the Z-radiation. Supposing, it were true that a bioactive radiation or a set of species informational signals really exists. From this assumption it follows, firstly, a circular chromosome or "prokaryotic STHE" antenna may be a receiver of Z-radiation. Secondly, before the beginning of cell cycle all cells are able to capture the own species signal. Thirdly, a cosmic signal, which was 
fixed on a DNA-wave, as on the physical bearer, can be realized in the phenotypic heterogenous organisms by means of intracellular informational system. This informational system has objects of class A, B and C, and this system as a whole possesses such properties as purposefulness, invariance, frailty, translatability, variability, efficiency, semantics, etc. $[4,5]$.

It is necessary to take into consideration the fact that the DNA-wave at the moment reception of cosmic signal already carries on itself the signal obtained by a cell before previous division. We will describe this type of information as the cellular DNA-information. A frequency of its oscillation is close to the frequency of cosmic signal, but is not identical to it. A prokaryotic cell is the open system and has a huge surface concerning its volume, which contacts with constantly varying environment (temperature, pressure, chemical compound, etc.). Biochemical processes and thermal motion of molecules into a cell also influence on the DNA-wave. Therefore DNA-information presents the modulated wave, which is formed as a result of the addition of two waves - a cosmic signal and a cellular DNAinformation (product of DNA-wave interactions with internal and external conditions during previous cell cycle). Since a part of information can be distorted or lost owing to the different noises and hindrances (a frailty property), its protection from the degradation is necessary for each generation of prokaryotic organisms. We think that reestablishment factor of genome may be only the invariable species signal from a cosmos.

In our model a cell is the self-oscillating system, in which a modulated DNA-wave carries out the undamped oscillations with a frequency close to the frequency of species cosmic signal. Therefore DNA-information can be considered as the build-in internal generator of electromagnetic oscillations or the intracellular heterodyne. The heterodyne allows the cell to configure a "prokaryotic STHE" at the reception of a cosmic signal of certain bandwidth. This means that a prokaryotic cell from physics positions presents the typical superheterodyne receiver. At the moment reception of signal, as a result of the mixing of two frequencies (cosmic and cellular), arises the intermediate frequency on which occurs the gain of species information signal coming from a cosmos.

A decoding is the last stage of a transformation of DNAinformation to the phenotypic heterogeneous individuals of one species. If we proceed from the wave nature of this process, the DNA-wave bearing the information signal, should generate the non-uniform electromagnetic biofield or the morphogenetic field by Weiss's terminology [32]. This field serves as an information matrix, on the basis of which the complex physical and biological form-building processes are began and species-specific shape and structure of unicellular organisms are acquired.

From the aforementioned it become obvious, the development of prokaryotic organisms in many respects depends from the information component of genome. A DNA-energy and a DNA-particle have an earthly origin, and a DNA-information has a cosmic and an earthly once. Information is not a material object, and the operators (circular chromosome, cell) are always and necessarily material objects. If a DNA-particle is most conservative system, a DNA-information depends on the environment. We suggest and describe a prokaryotic cell as the superheterodyne receiver, which carries out a reception and a transfer of cosmic species signal in the species specificity of microorganisms. From physics position a prokaryotic cell and it genetic apparatus able to provide all these processes. The frame antenna of "prokaryotic STHE" type characterizes a very good selectivity and ability to detect even weak signals of certain frequency.

\section{Conclusions}

1) A cell is organized in three independent and interlinked planes, and, accordingly, has three programs of development - energetic, corpuscular and informational, which in prokaryotic organisms are pawned and realized during one cell cycle.

2) All planes are the attributes of the DNA molecule (particle and wave) in three its hypostasis - DNAenergy, DNA-particle and DNA-information. DNAenergy and DNA-information have a wave nature, and DNA-particle - the corpuscular ones. DNA-energy and DNA-particle have an earthly origin, and DNAinformation - a cosmic and an earthly nature. They are the equal in their significance. To divide and join these three essences of genome is not possible.

3) Central place belongs to the DNA-energy. A cell life is completely subordinated to the creation of conditions, which is necessary for the preservation and support of the continuous work of biological harmonic oscillator as the physical body. In its turn, all its attributes provides feedback with DNA-particle and DNAinformation as: 1) a oscillator as the source of free and total energy; 2) a standing wave as the transport system for replication and transcription reactions; 3) a selfoscillation system for modulation of DNA-wave oscillations; 4) a biofield as the matrix for creation of morphogenetic field, etc.

4) A trinity principle represents energy, matter and information unity, which is realized on the basis of DNA molecule (particle and wave) at the cellular level, and is the emergent property of organism and species levels. Therefore only a cell may be the elementary and indivisible unit of living matter.

\section{References}

[1] Shredinger, E., 1972. What is Life? From the Point of View of Physics. Atomizdat, Moscow.

[2] Shannon, C. E., 1949. A Mathematical Theory of Communication. Urbana, Illinois, University of Illinois Press. 
[3] Ashby, W. R., 1956. An Introduction in Cybernetics. Chapman \& Hall, London.

[4] Korogodin, V. I., 1983. The definition of "information" notion and the possibility of its use in biology. Russian J. Biophysics. XXVIII (1): 171-177.

[5] Korogodin, V. I. and Korogodina, V. L., 2000. Information as the basis of life. Pupl. "Fenix", Dubna.

[6] Thilms, L., 2012. Thermodynamics $\neq$ Information Theory: Science's Greatest Sokal Affair. J. Thermodynamics, 8(1): 1120.

[7] Nina F. Kuznetsova, 2013. Corpuscular-wave Nature and Wave Properties of Plant Cells. Nova Science Publ., Inc. New York.

[8] Davis, B. D., Dubecco, R., Eisen, N. N. and Ginsberg, H. S., 1990. Microbiology. $4^{\text {th }}$ Ed. Lippicott Comp., Philadelphia.

[9] Karp, G., 1996. Cell and Molecular Biology: Concepts and Experiments. John Willey and Sons Inc., New York.

[10] Chentsov, Yu. S., 2004. Introduction to the Cell Biology. Akademkniga, Moscow.

[11] Sakura, Y, Kato, 2011. Archaea: Structure, Habitats and Ecological Significance. Nova Science Publ., Inc. New York.

[12] Rob Phillips, Jane Kondev, Julie Theriot and Herman G. Garci, 2012. Physical Biology of the Cell. $2^{\text {nd }}$ Ed. Garland Science, Taylor \& Francis Group, London, New York.

[13] Alison Snape, Despo Papachristodoulou, William H. Elliot and Daphne C. Elliot, 2014. Biochemistry and Molecular Biology. Oxford University Pbl., Oxford, United Kingdom.

[14] Bruce Alberts, Alexander Johnson, Julian Lewis, David Morgan, Martin Raff, Keith Roberts and Peter Walter, 2014. Molecular Biology of the Cell, $6^{\text {th }}$ Ed. Chaffey, N (Ed.), Garland Science, Taylor \& Francis Group, New York.

[15] Volkenshtein, M. V., 1983. Theoretical Biophysics. Nauka, Moscow.

[16] Gurvich, A. G., 1944. Theory of Biological Field. Sovetskay nauka, Moscow.

[17] Mazia, D., 1961. Mitosis and the Physiology of Cell Division. Ed. J. Brachet \& A. Mirsky V. III. Academic Press, New York and London.
[18] Hegel, G. W. F., 1932. Works. V. 9. Partiynoe delo, Moscow.

[19] Gusev, V. A., 2001. About the Energy Source for Preserve the Viability and Amplification of Heterotrophic Organisms under the Conditions of Substrate Starvation. I. Formulation of Hypothesis. Russian J. Biophysics, 46: 862-868.

[20] Riegel, J. A., 1967. Energy, Life and Organism. Mir, Moscow.

[21] Benjamin A. Pierce, 2014. Genetics: A Conceptual Approach. $5^{\text {th }}$ Ed. W. H. Freeman and Co Ltd, New York, United States.

[22] Rees, A. R. and Sternberg, M. J. E. 2002. Introduction to Molecular Biology: From Cells to Atoms. Mir, Moscow.

[23] Drake, J. W., Charlesworth, B., Charlesworth, D. and Crow, J. F., 1998. Rates of Spontaneous Mutation. Genetics, 148: 1667-1686.

[24] Fox, S. and Dose, K., 1972. Evolution and the Origin of Life. Dekker, New York.

[25] Kaznacheyev, V. P. and Mikhailova, L. P., 1981. Super-weak Radiation in the Intercellular Interactions. Nauka SO AN, Novosibirsk.

[26] Shcherbakov, V. P., 2005. Evolution as Resistance to Entropy. I. The Mechanisms of Species Homeostasis. Biology Bulletin Reviews. 66: 195-211.

[27] Denbigh, K. G., 1989. Note on Entropy, Desorder and Desorganization. British Journal of Phylosophy and Science. 40: 423-432.

[28] Wiener, N., 1983. Cybernetics or Control and Communication in the Animal and the Machine. Main editorial office for foreign countries "Science", Moscow.

[29] Wicken, J. S., 1979. The Generation of Complexity in Evolution: a Thermodynamic and Information-theoretical Discussion. J. Theor. Biol., 77(3): 349-365.

[30] Vernadsky, V. I., 1967. Biosphere. Mysl, Moscow.

[31] Chizhevsky, A. L., 1972. Certain Cosmic Communications Terrestrial Biosphere. Populated cosmos. Nauka, Moscow.

[32] Weiss, PA., 1928. Morphodynamische Feldtheorie und Genetik. Zeitschrift für induktive Abstammungs - und Vererbungslehre, 2: 1567-1574. 